\title{
High-Resolution X-Ray Spectroscopy with AXAF
}

\author{
G. Fabbiano
}

Harvard-Smithsonian Center for Astrophysics, 60 Garden St., Cambridge, MA 02138, USA

\begin{abstract}
This paper is a very short description of the Advanced $X$ ray Astronomy Facility $(A X A F)$ and its spectral capabilities. A more detailed description can be found at the WWW location http://heawww.harvard.edu/asc/SIN/SIN.html.
\end{abstract}

\section{AXAF}

The Advanced X-ray Astronomy Facility (AXAF) is scheduled to be launched by NASA on 1998 August 27. The first call for proposals (NRA) is planned for 1997 September 1.

$A X A F$ will observe the sky in a wide $\mathrm{X}$-ray band (0.1-10 keV). The angular resolution of $A X A F$ is $0^{\prime \prime} .5$ (FWHM). $A X A F$ will then return extremely good images of the X-ray sky, and because of its small beam size will be able to achieve very deep, sensitive images.

$A X A F$ is not only an imaging telescope, it also carries medium and highresolution $\mathrm{X}$-ray spectrometers.

\subsection{ACIS}

The $A X A F$ CCD Imaging Spectrometer (ACIS) is one of the two focal-plane detectors and achieves moderate spectral resolution with $E / \Delta E \approx 10-60$, increasing to high energies. Its effective area is a factor of $\sim 2$ larger than that of $A S C A$ (in the currently used one-CCD observing mode) between 2 and $10 \mathrm{keV}$. It is much superior at lower energies, going from $800 \mathrm{~cm}^{2}$ at $1 \mathrm{keV}$ to $200 \mathrm{~cm}^{2}$ down to $0.2-0.3 \mathrm{keV}$. Because the $A X A F$ beam size is $10^{4}$ times smaller than that of $A S C A$, background is negligable for point sources. The imager field of view is $16^{\prime} \times 16^{\prime}$.

\subsection{Gratings}

There are two sets of gratings in $A X A F$, whose dispersed spectrum will be imaged onto each of the two focal-plane detectors, the High Resolution Camera (HRC) and ACIS. The spectral resolution of the gratings is $E / \Delta E \approx 200-3000$, increasing at lower energies. No slit is available. This limits the use of gratings to point-like sources, and care in the choice of the satellite roll angle will have to be taken when observing crowded fields. 
LETG The Low Energy Transmission Grating (LETG) spectra are imaged on the HRC. The effective area ranges between $10 \mathrm{~cm}^{2}$ and $40 \mathrm{~cm}^{2}$ between 0.1 and $5 \mathrm{keV}$. A two-facet reflection filter (the Drake flat) will be used for order separation.

HETG The High Energy Transmission Grating (HETG) spectra are imaged on the ACIS. Since the ACIS is itself a spectrometer, this provides an immediate advantage for grating spectral-order separation. The effective area ranges from $10 \mathrm{~cm}^{2}$ at $0.5 \mathrm{keV}$ to $100 \mathrm{~cm}^{2}$ on average between 1 and $5 \mathrm{keV}$.

\section{For More Information}

More information on $A X A F$ and its instruments can be found at the following World Wide Web addresses:

http://hea-www.harvard.edu/asc/axaf-welcome.html

http://hea-www.harvard.edu/asc/SIN/SIN.htmI 